\title{
Atomic Oxygen Adsorption on Core-shell Ni@Pt and Pure Pt Nanoparticles
}

\author{
Ferensa OEMRY*1, Hiroshi NAKANISHI*1, Hideaki KASAI*1,*2, \\ Hiroyoshi MAEKAWA*3, Kazuo OSUMI ${ }^{* 3}$ and Kaoru SATO*3 \\ ${ }^{*}$ Division of Precision Science \& Technology and Applied Physics, Graduate School of Engineering, Osaka University, \\ Suita, Osaka 565-0871, Japan \\ ${ }^{* 2}$ Center for Atomic and Molecular Technologies, Osaka University Suita, Osaka 565-0871, Japan \\ ${ }^{*}$ Isuzu Advanced Engineering Center, Ltd., Fujisawa, Kanagawa 252-0881, Japan
}

(Received August 1, 2013, Accepted May 8, 2014)

\begin{abstract}
Atomic oxygen adsorption on core-shell Ni@Pt and pure Pt nanoparticles composed of 55 atoms each is investigated using first-principles calculations. Results show that 5d-5d hybridization between Pt(core) and Pt(shell) in pure Pt cluster demonstrates both attractive and repulsive bonding characters depending on oxygen adsorption site. However, for Ni@Pt cluster, $3 \mathrm{~d}-5 \mathrm{~d}$ hybridization between $\mathrm{Ni}$ (core) and $\mathrm{Pt}($ shell) in the presence of oxygen always impose repulsive bonding character, which in turns, weakens the bonding at $\mathrm{Ni} / \mathrm{Pt}$ interface.
\end{abstract}

\section{Introduction}

Current diesel oxidation catalyst (DOC) ${ }^{1-3)}$ that employs smaller Pt nanoparticles is still experiencing several drawbacks because those nanoparticles are notoriously susceptible to oxygen poisoning ${ }^{4)}$, deformation ${ }^{5)}$, and sintering processes ${ }^{6,7)}$. In recent years, heterogeneous catalyst such as $\mathrm{NiPt} / \mathrm{Al}_{2} \mathrm{O}_{3}$ has been reported to show better catalytic reactivity in dry reforming of methane $(\mathrm{DRM})^{8,9)}$, ethanol steam reforming for hydrogen production ${ }^{10,11)}$ and hydrogenation of maleic anhydride $(\mathrm{MA})^{12)}$. Experimental studies have also demonstrated that the catalyst can operate at higher temperatures, which is mandatory for the operational working temperature of DOC during catalytic oxidation. Therefore, the catalyst has greater chance to improve and enhance the current catalytic oxidation of DOC. In previous study ${ }^{13)}$, we have investigated the possibility of utilizing core-shell $\mathrm{Ni@Pt} \mathrm{nanoparticles} \mathrm{as} \mathrm{prospective} \mathrm{catalyst} \mathrm{candidate} \mathrm{in}$ DOC. One important result in that study is that the substitution of $1 \mathrm{~nm}$ Pt nanoparticles with core-shell Ni@Pt nanoparticles of similar size could weaken Pt-O bonds. The result suggests that core-shell Ni@Pt nanoparticles can resolve oxygen poisoning issue that affects turn over frequency (TOF) of $\mathrm{NO}$ oxidation on $1 \mathrm{~nm} \mathrm{Pt}$ nanoparticles ${ }^{14)}$. In addition, adsorbed oxygen-induced cluster reconstruction phenomenon that was found in core-shell Ni@Pt and pure Pt nanoparticles has also been elaborated. In correlation to the observation made by EXAFS measurements ${ }^{15,16)}$, our calculation results qualitatively provide similar general trend where the amplitude of the first nearest neighbor (1NN) Pt-Pt bond distribution of surface atoms decreases as more oxygen adsorbed on the nanoparticles.

There are still some lacks of knowledge about how this cluster reconstruction might affect the bonding at $\mathrm{Ni} / \mathrm{Pt}$ interface, whether it would strengthen or weaken the bonding is still unknown. This concern arises from the fact that oxygen do not only locally induce surface atoms rearrangement on the surrounding adsorption site but also globally affect the connecting bonds of all nanoparticle atoms ${ }^{13)}$. Similar phenomenon have also been observed in oxidized $\mathrm{Na}_{55}$ nanoparticle ${ }^{17)}$. Furthermore, an early investigation on $\mathrm{Pt}(111)$ surface has reported that adsorbed oxygen can strengthen and weaken the bonding between subsurface and surface Pt atoms by means of $\mathrm{d}$ electron frustration ${ }^{18)}$. Therefore, in this work, we perform density functional theory (DFT) calculations in order to scrutinize the stability of Ni-Pt bonds in the presence of oxygen in core-shell Ni@Pt nanoparticles. In the case, if Ni-Pt bonds are proven to be weakened, it should be clarified whether it undermines the effectiveness of compressive strain effect ${ }^{19)}$ on Pt-Pt bonds in $\mathrm{Ni}$ @ Pt nanoparticles to weakly attract oxygen or not. For reference, (non-alloyed) pure Pt cluster is used for sideby-side comparison. Moreover, this work is also meant to check the applicability of d-electron frustration model to be implemented on alloyed NiPt metals. In general, we mainly focus our discussion on two points related to Pt-O bonds formation on Ni@Pt cluster: (1) Role of alloying and geometric relaxation in adjusting oxygen adsorption energy; (2) The effect of platinum oxide formation on the bonding at $\mathrm{Ni} / \mathrm{Pt}$ interface and its direct implication to the interface.

\section{Computational Methods}

To construct $1 \mathrm{~nm}$ nanoparticles, we adopted the socalled magic cluster (i.e., clusters containing complete shell of atoms) with size of 55 atoms ${ }^{20,21)}$ and found that Mackay icosahedron to be the most stable structure among other 55-atom Pt cluster isomers. From previous computational study, Wang et al. ${ }^{21)}$ through their seminal DFT study has confirmed that the alloyed Ni-Pt cluster using 55 atoms is energetically more favorable to accommodate Pt-enriched surface. This allowed us to construct Ni@Pt cluster where 42 out of 55 atoms are positioned on the cluster surface.

All the calculations were performed with the VASP 
program $^{22-24)}$ using the generalized gradient approximation (GGA) with Perdew-Burke-Ernzerhof (PBE) ${ }^{25)}$ for the exchange correlation functional and the projector augmented-wave (PAW) method using a plane-wave cutoff energy of $400 \mathrm{eV}$. Using one k-point sampling, the energies were converged to $1 \mathrm{meV} /$ atom and ionic relaxations were allowed until the absolute value of force on each atom was below $0.02 \mathrm{eV} / \AA$. The clusters were simulated in a periodic cubic supercell with a size of $25 \AA$ sides, ensuring a sufficient vacuum region to separate the clusters and their images. For geometry optimization, two calculation schemes are carried out. For rigid cluster calculation, oxygen atom was relaxed and all atoms in the clusters were fixed. While for relaxed cluster case, oxygen and all atoms in the clusters, except one fixed atom in the center, were fully relaxed. Oxygen adsorption energy $\left(E_{\text {ad }}\right)$ was calculated from the total energy difference between the system with adsorbate $\left(E_{\text {cluster-O }}\right)$, isolated cluster $\left(E_{\text {cluster }}\right)$, and isolated oxygen $\left(E_{\mathrm{O}}\right)$ relative to $1 / 2 \mathrm{O}_{2}$ in gas phase as given by the equation, $E_{\mathrm{ad}}=$ $E_{\text {cluster-O }}-\left(E_{\text {cluster }}+E_{\mathrm{O}}\right)$. The oxygen adsorption energies for rigid and relaxed clusters are obtained from previous study ${ }^{13)}$.

\section{Results and Discussions}

\subsection{Role of alloying and geometric relaxation effects}

Figure 1(a) and 1(b) show the optimized core-shell Ni @Pt and pure Pt clusters, respectively, where the surface (shell) atoms arrangement gives 20 identical equilateral triangular faces and all of them share similar (111) facet surface. The average Pt-Pt bond distances on the surface of core-shell Ni@Pt and pure Pt clusters are 2.68 $\AA$ and $2.79 \AA$, respectively, which closely match the values obtained from X-ray diffraction (XRD) data of $2.70 \AA$ and $2.77 \AA 26,27$. Furthermore, there are two distinguishable Pt atoms on the surface as seen in Fig. 1(a) and 1(b), indicated by A and B labels that refer for atoms with 6 and 8 coordination numbers, respectively. The average bond distances for A-B and B-B bonds in Ni@Pt cluster are $2.63 \AA$ and $2.73 \AA$, respectively, while the ones in pure Pt cluster are $2.72 \AA$ and $2.86 \AA$. It is clear that all shell Pt-Pt bonds in Ni@Pt cluster are contracted due to the $\mathrm{Ni}$ core atoms.

Here, we consider six adsorption sites as indicated in Fig. 1(c). The oxygen adsorption energies are summarized in Table 1 for both rigid and reconstructed clusters. For all sites, oxygen is found to be adsorbed on pure Pt cluster slightly stronger than on Ni@Pt cluster. The trend of weaker oxygen adsorption energy in Ni@Pt cluster is mainly caused by strong $3 \mathrm{~d}-5 \mathrm{~d}$ interaction between Ni(core) and Pt(shell) atoms (see Fig. 1(d)). This trait can be easily observed from the shifting of some $5 \mathrm{~d}$ states of $\mathrm{Pt}$ atoms (A and $\mathrm{B}$ ) to lower energy region (as pointed out by the arrows) and leaving less available Pt$5 \mathrm{~d}$ states that could interact with $\mathrm{O}-2 \mathrm{p}$ states. Hence, the shell Pt atoms in Ni@Pt cluster could not bind oxygen as strong as those atoms in pure Pt cluster (alloying effect).

Furthermore, the geometric structure of these two clusters during Pt-O bonds formation is not completely rigid although, following oxygen adsorption, there is no noticeable structural change. However, the stronger oxygen adsorption energy found in reconstructed cluster

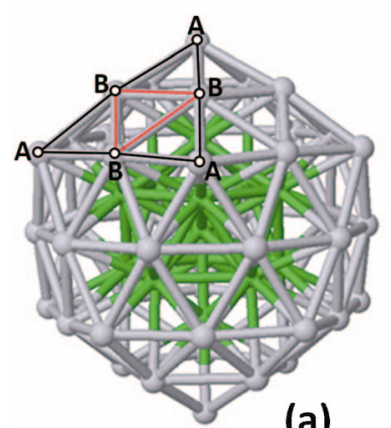

(a)
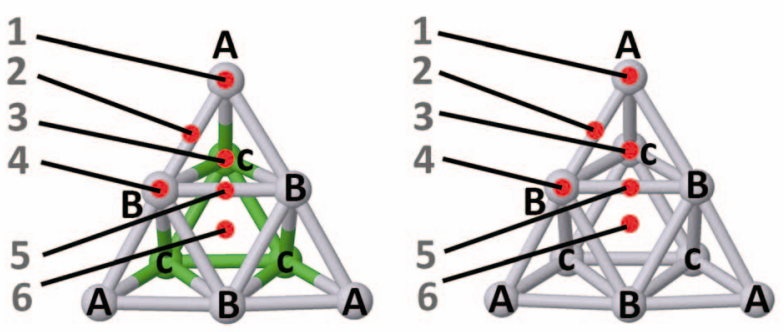

(c)

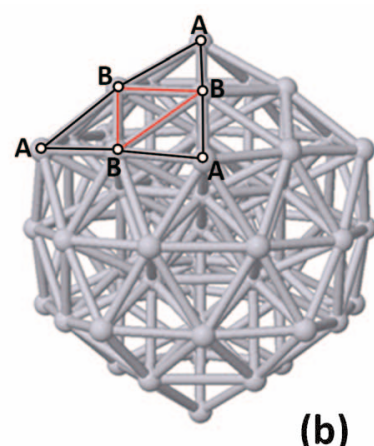

(b)
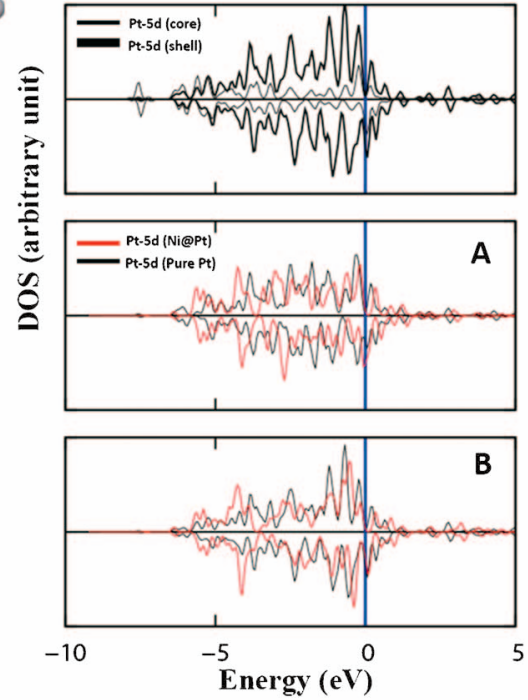

(d)

Fig. 1 Optimized structures of core-shell Ni@Pt (a) and pure Pt (b) clusters where the designated oxygen adsorption sites (c) are shown by numbers from 1 to 6 on small representative structure of each cluster. The projected density of states (PDOS) of both clusters (d) is provided for total shell and core atoms (two topmost panels) and single atoms A and B (two bottom panels). Gray and green spheres are corresponding to platinum and nickel atoms. 
Table 1 Oxygen adsorption energy on various designated adsorption sites on Ni@Pt and pure Pt clusters [13]. The unit is given in $\mathrm{eV}$.

\begin{tabular}{ccccc}
\hline \hline $\begin{array}{c}\text { Cluster } \\
\text { type }\end{array}$ & $\begin{array}{c}\text { Site } \\
\text { number }\end{array}$ & $\begin{array}{c}\text { Site } \\
\text { position }\end{array}$ & $\begin{array}{c}\text { Relaxed } \\
\text { cluster }\end{array}$ & $\begin{array}{c}\text { Rigid } \\
\text { cluster }\end{array}$ \\
\hline $\mathrm{Ni} @ \mathrm{Pt}$ & 1 & vertex A & -4.06 & -3.96 \\
& 2 & bridge & -4.51 & -4.18 \\
& 3 & hcp-like & -4.51 & -4.19 \\
& 4 & vertex B & -3.45 & -3.06 \\
& 5 & bridge B & \multicolumn{2}{c}{ unstable } \\
& 6 & fcc-like & -4.22 & -3.91 \\
\hline Pure Pt & 1 & vertex A & -4.27 & -4.11 \\
& 2 & bridge & -4.51 & -4.30 \\
& 3 & hcp-like & -4.61 & -4.28 \\
& 4 & vertex B & -3.76 & -3.58 \\
& 5 & bridge B & \multicolumn{2}{c}{ unstable } \\
& 6 & fcc-like & -4.55 & -4.14 \\
\hline
\end{tabular}

rather than that obtained from rigid cluster indicates that geometric relaxation has occurred in the clusters. According to $\mathrm{Lu}$ and Henkelman ${ }^{28}$, this geometric relaxation which is induced by oxygen stabilizes Pt-O bonds formation and reduces the catalytic activity of Pt-based nanoalloys. Hence, geometric relaxation could undermine the effectiveness of core $\mathrm{Ni}$ atoms in rendering contracted Pt-Pt bonds on the surface to weakly bind oxygen. This explains why oxygen adsorption energies on core-shell Ni@Pt cluster have small energy gap with respect to those of pure Pt cluster. However, one should remember that both alloying and geometric relaxation effects do not dictate the oxygen adsorption site preference in the clusters because the geometric structure of the clusters is still dominant factor in determining the most preferable adsorption site for oxygen. This similar observation has also been confirmed for oxygen adsorption site preference on 1 and $2 \mathrm{~nm} \mathrm{Pt}$ nanoparticles with respect to $\mathrm{Pt}(111)$ surface $^{29)}$.

\subsection{The mechanism of bond weakening process at $\mathrm{Ni} / \mathrm{Pt}$ interface}

\subsubsection{Ni-Pt bond weakening and Pt-Pt bond strength- ening}

To understand the mechanism of oxygen adsorption in disrupting the bonding at core/shell interface, schematics of three selected adsorption sites are obtained by slicing through atoms with A-c-B-c-A labels (see Fig. 1(c)) and illustrated in Fig. 2(a)-2(c). For vertex A and bridge sites case, A-designated shell $\mathrm{Pt}$ and c-designated core $\mathrm{Ni}$ (or $\mathrm{Pt}$ ) atoms are distinguished by assigning them a number to indicate which $\mathrm{Pt}$ and/or Ni atom(s) are involved in the bond weakening/strengthening process at the core/shell interface.

For vertex A site case in pure Pt cluster (the two upper panels on the left side of Fig. 3), following oxygen adsorption, strong $2 \mathrm{p}-5 \mathrm{~d}$ hybridization between oxygen and shell $\operatorname{Pt}\left(\mathrm{A}_{1}\right)$ atoms generates two distinct sequential peaks of bonding and anti-bonding regions. The $5 \mathrm{~d}$ electrons of core $\mathrm{Pt}\left(\mathrm{c}_{1}\right)$ atom are also redistributed to occupy the same energy levels that adopted by $5 \mathrm{~d}$ electrons of
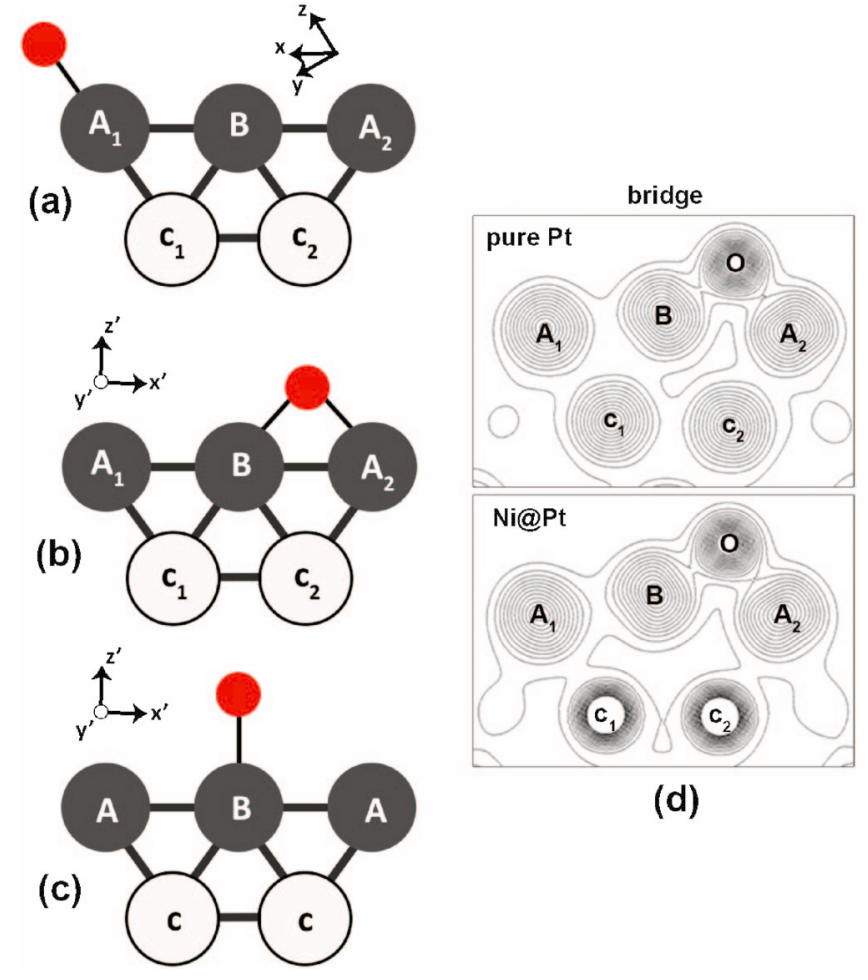

(d)

Fig. 2 Schematics of three selected adsorption sites: (a) vertex A, (b) bridge, and (c) vertex $\mathrm{B}$ which obtained by slicing through atoms with A-c-B-c-A labels. Red, black, and white spheres are representing oxygen, shell Pt, and core atoms, respectively. The $y$-axis in bridge and vertex $B$ sites is pointing inward. Charge density distribution in $\mathrm{Ni@Pt}$ and pure $\mathrm{Pt}$ clusters for bridge site case (d) is gained using isosurface interval $4.5 \times 10^{-3} \mathrm{e}^{-/} \AA^{3}$.

$\operatorname{Pt}\left(\mathrm{A}_{1}\right)$ atom. As the result, the $5 \mathrm{~d}$ electrons of both $\mathrm{Pt}$ atoms that make contribution to constructive bonding of $\operatorname{Pt}\left(\mathrm{c}_{1}\right)-\operatorname{Pt}\left(\mathrm{A}_{1}\right)$ bond are shifted to lower energy level while the electrons that build anti-bonding interaction are displaced above the Fermi level. This indicates that $\operatorname{Pt}\left(\mathrm{c}_{1}\right)$ $\operatorname{Pt}\left(\mathrm{A}_{1}\right)$ bonds are strengthened. Hence, the $5 \mathrm{~d}-5 \mathrm{~d}$ hybridization between $\operatorname{Pt}\left(\mathrm{c}_{1}\right)$ and $\operatorname{Pt}\left(\mathrm{A}_{1}\right)$ atoms generates more attractive bonding character upon oxygen presence.

As for the Ni@Pt cluster case (the two bottom panels on the left side of Fig. 3), the 2p-5d hybridization provides electron distribution profile similar to the one exhibited in pure Pt cluster. However, the $3 \mathrm{~d}$ electrons of $\mathrm{Ni}\left(\mathrm{c}_{1}\right)$ atom are unexpectedly shifting into the interval energy region $[-3.3,-0.35]$. Since the majority distribution of $\mathrm{Ni}\left(\mathrm{c}_{1}\right)-3 \mathrm{~d}$ and $\operatorname{Pt}\left(\mathrm{A}_{1}\right)-5 \mathrm{~d}$ electrons in post-oxygen adsorption state are no longer staying at the same energy range, it can be argued that $\mathrm{Ni}\left(\mathrm{c}_{1}\right)-\operatorname{Pt}\left(\mathrm{A}_{1}\right)$ bond has been weakened. Therefore, the $3 \mathrm{~d}-5 \mathrm{~d}$ hybridization of the Ni$\mathrm{Pt}$ bond has become more repulsive after the Pt-O bond formation. Partial charge density distribution plots in Fig. 4 further rationalize this bond weakening process in $\mathrm{Ni} @ \mathrm{Pt}$ cluster as follows. The weakening $\mathrm{Ni}\left(\mathrm{c}_{1}\right)-\operatorname{Pt}\left(\mathrm{A}_{1}\right)$ bond is compensated by increasing bonding strength of $\mathrm{Ni}\left(\mathrm{c}_{1}\right)-\mathrm{Ni}\left(\mathrm{c}_{3}\right)$ bond due to electron transfer from $\mathrm{Ni}\left(\mathrm{c}_{3}\right)$ to $\mathrm{Ni}\left(\mathrm{c}_{1}\right)$ atom as seen in the plots at energy range [-3.3, -2.3 . Some parts of these electrons also contribute to 


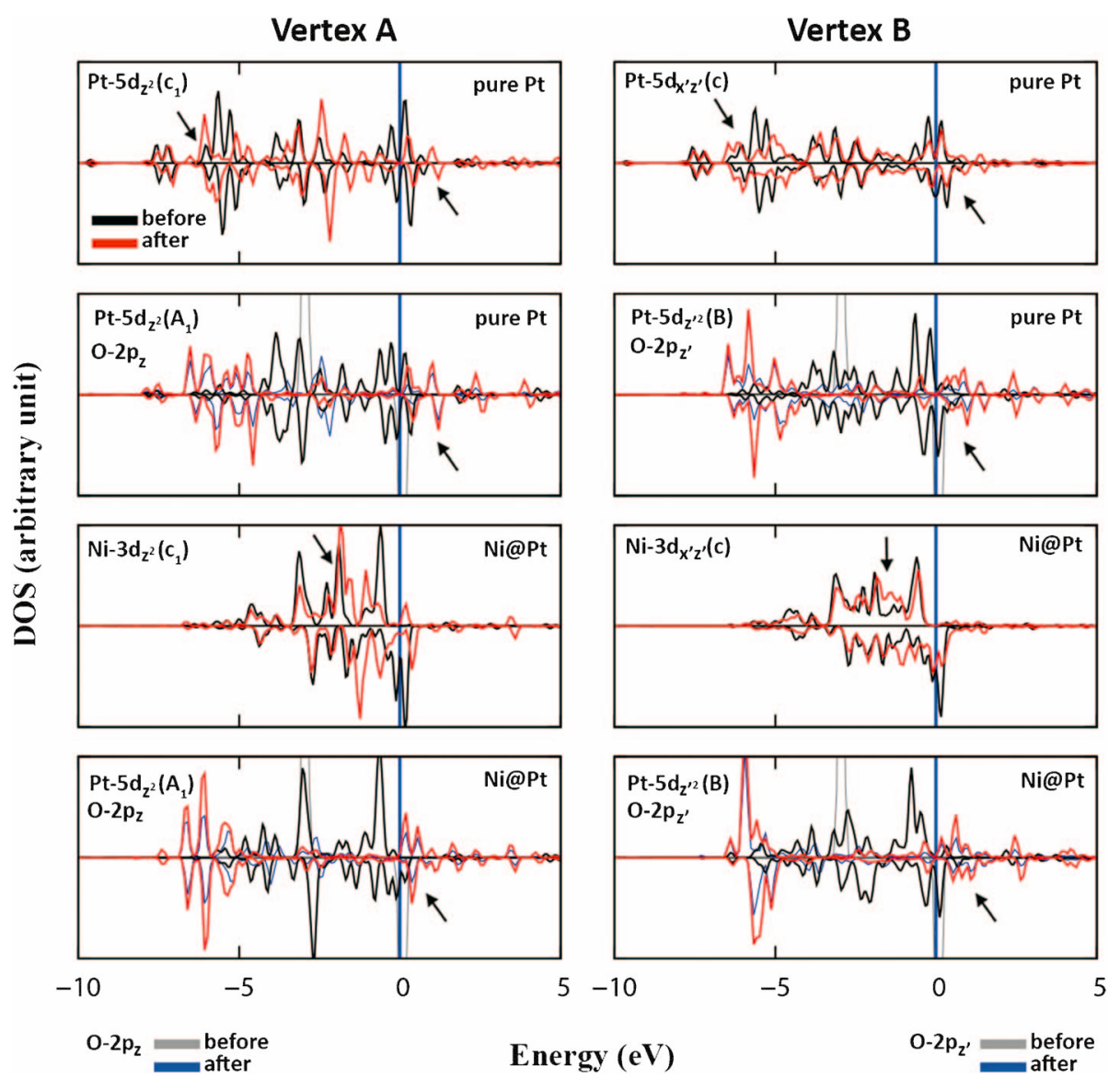

Fig. 3 Projected density of states (PDOS) describe bonding interactions between shell Pt and core Pt (or Ni) atoms in vertex A (left) and B (right) sites. Solid black (gray) and red (blue) lines correspond to Pt-5d states or Ni-3d (O-2p) states before and after oxygen adsorption takes place. The Fermi level is taken at zero energy level. The arrows serve as eye guidance to indicate $3 \mathrm{~d}$ and $5 \mathrm{~d}$ states final displacement positions after oxygen is adsorbed.
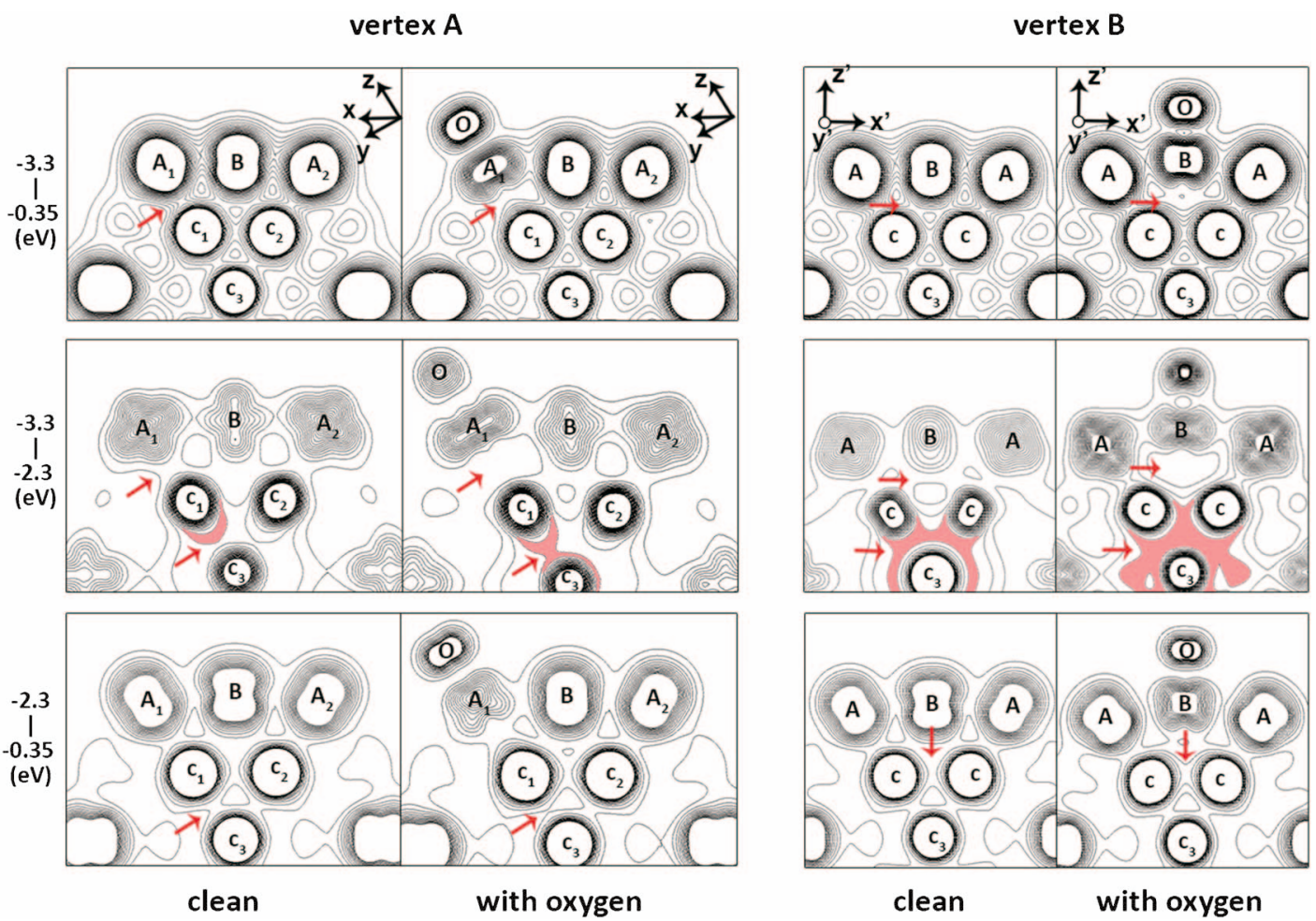

Fig. 4 Contours of partial charge density distribution in Ni@Pt cluster for the case of vertex A and vertex B sites. The partial charge density plots at interval energies $[-3.3,-0.35]$ and $[-3.3,-2.3]$ are drawn using isosurface interval $4.5 \times 10^{-3} \mathrm{e}^{-/} \AA^{3}$, while the plots at interval energy level $[-2.3,-0.35]$ is obtained using isosurface interval $7 \times 10^{-3} \mathrm{e}^{-/} \AA^{3}$ for better clarity and contrast. 
the increasing repulsive (anti-bonding) interaction between these two atoms as emphasized by the charge density plots at $[-2.3,-0.35]$.

For vertex B site case, different cartesian coordinate system $\left(x^{\prime}, y^{\prime}, z^{\prime}\right)$ is used as described in Figs. 2(c) and 3. When oxygen is adsorbed on pure Pt cluster, the $\mathrm{Pt}(\mathrm{c})-5 \mathrm{~d}$ electrons will undergo similar displacement that is experienced by the $\mathrm{Pt}(\mathrm{c} 1)-5 \mathrm{~d}$ electrons in vertex $\mathrm{A}$ case. In similar way, some of Ni(c)-3d electrons of Ni@Pt cluster are shifted to the interval energy region $[-3.3,-0.35]$ and this depletes the charge density in the midpoint region of $\mathrm{Ni}(\mathrm{c})-\mathrm{Pt}(\mathrm{B})$ bonds as clearly seen in the plots of Fig. 4. The event of weakening $\mathrm{Ni}(\mathrm{c})-\mathrm{Pt}(\mathrm{B})$ bonds in $\mathrm{Ni} @ \mathrm{Pt}$ cluster is subsequently followed by the expansion of contour area of charge density (shaded region) that envelops $\mathrm{Ni}(\mathrm{c})$ and $\mathrm{Ni}\left(\mathrm{c}_{3}\right)$ atoms. Through the depiction of partial charge density plot in Fig. 4 at energy range $[-2.3$, $-0.35]$, it is found that the $\mathrm{Ni}(\mathrm{c})-\mathrm{Ni}(\mathrm{c})$ bond has been strengthened instead of the $\mathrm{Ni}(\mathrm{c})-\mathrm{Ni}(\mathrm{c} 3)$ bonds since there is a rise of charge density accumulation in the midpoint region of $\mathrm{Ni}(\mathrm{c}) \mathrm{Ni}(\mathrm{c})$ bond axis after oxygen adsorption.

\subsubsection{Effect of $\mathrm{Pt}$ oxide formation on $\mathrm{Ni} / \mathrm{Pt}$ interface and its direct implication}

For the bridge site case (see Fig. 2(b)), one oxygen atom is bonded to two $\mathrm{Pt}$ atoms. Another interesting thing is that oxygen adsorption energy in this site is similar for both relaxed clusters as consequence of the geometric relaxation effect. In order to answer why both clusters can bind oxygen at equal Pt-O bonding strength, the bonding at $\mathrm{Ni} / \mathrm{Pt}$ and $\mathrm{Pt}$ (core)/Pt(shell) interfaces will be further evaluated. Based on the PDOS analysis in Fig. 5, the attractive bonding interaction at core/shell interface in pure $\mathrm{Pt}$ cluster is represented only by $\mathrm{Pt}\left(\mathrm{c}_{1}\right)-\mathrm{Pt}(\mathrm{B})$ bond, while the repulsive ones are given by $\operatorname{Pt}\left(\mathrm{c}_{2}\right)-\mathrm{Pt}(\mathrm{B})$ and $\mathrm{Pt}\left(\mathrm{c}_{2}\right)-\mathrm{Pt}\left(\mathrm{A}_{2}\right)$ bonds. These bonds with respect to their equilibrium bond lengths (without oxygen) are readjusted by $-0.06 \AA, 0.17 \AA$, and $0.04 \AA$, respectively. The contracted $\mathrm{Pt}\left(\mathrm{c}_{1}\right)-\mathrm{Pt}(\mathrm{B})$ bond (minus sign) is a good indication that the bond has been strengthened. On the other hand, all bonding interactions at Ni/Pt interface in Ni@Pt cluster are imposing repulsive (anti-bonding) interaction as demonstrated by $\mathrm{Ni}\left(\mathrm{c}_{1}\right)-\mathrm{Pt}(\mathrm{B}), \mathrm{Ni}\left(\mathrm{c}_{2}\right)-\mathrm{Pt}(\mathrm{B})$, and $\mathrm{Ni}\left(\mathrm{c}_{2}\right)-\operatorname{Pt}\left(\mathrm{A}_{2}\right)$ bonds. All of these bonds are being stretched by $0.21 \AA, 0.38 \AA$, and $0.06 \AA$ respectively.

PDOS in Fig. 5 confirms that core $\mathrm{Ni}\left(\mathrm{c}_{1}\right)$ and $\mathrm{Ni}\left(\mathrm{c}_{2}\right)$ atoms in $\mathrm{Ni@Pt} \mathrm{cluster} \mathrm{experience} \mathrm{an} \mathrm{increase} \mathrm{of} \mathrm{anti-}$ bonding interaction, following the oxygen adsorption, while in pure Pt cluster this anti-bonding interaction can only be found in $\operatorname{Pt}\left(\mathrm{c}_{2}\right)$ atom. As additional information, $\operatorname{Pt}\left(\mathrm{c}_{2}\right)-\operatorname{Pt}\left(\mathrm{A}_{2}\right)$ and $\mathrm{Ni}\left(\mathrm{c}_{2}\right)-\operatorname{Pt}\left(\mathrm{A}_{2}\right)$ bonds are noticed to have the shortest bond elongation values with respect to those of $\mathrm{Pt}\left(\mathrm{c}_{2}\right)-\mathrm{Pt}(\mathrm{B})$ and $\mathrm{Ni}\left(\mathrm{c}_{2}\right)-\mathrm{Pt}(\mathrm{B})$ bonds. The reason is that the anti-bonding interaction in the later bonds occurs in similar atomic orbital $\left(\mathrm{d}_{\mathrm{xz}}\right)$ while the former bonds are resulted from different atomic orbitals interaction $\left(\mathrm{d}_{\mathrm{xz}} \mathrm{vs} \mathrm{d}_{\mathrm{z}}^{2}\right)$. Thus, their anti-bonding interaction is

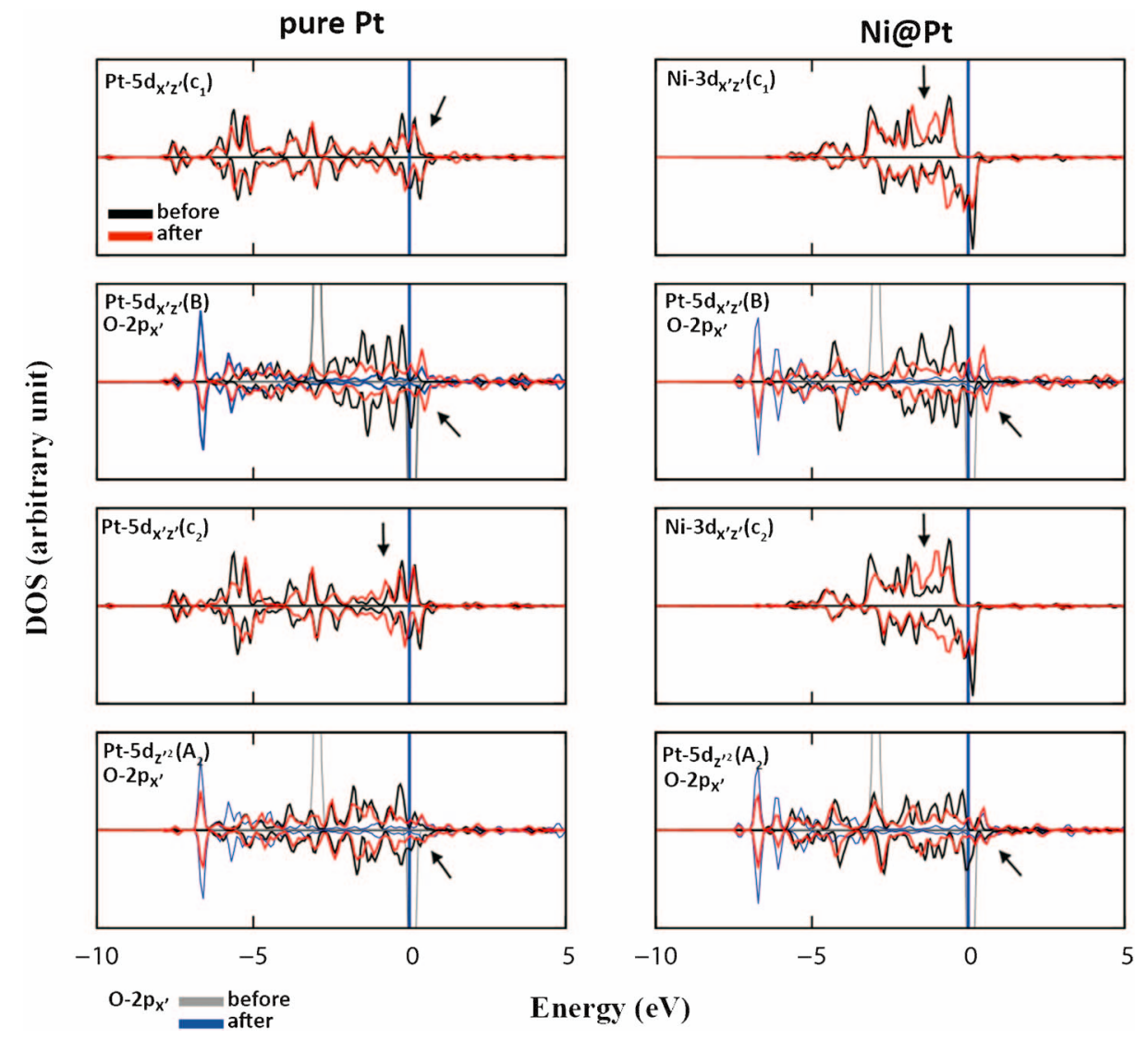

Fig. 5 Projected density of states (PDOS) of bonding interactions between shell Pt and core Pt (or Ni) atoms in bridge site case. For details, please see caption of Fig. 3. 
less effective.

The bond strengthening process that occurs in $\mathrm{Pt}\left(\mathrm{c}_{1}\right)-\mathrm{Pt}(\mathrm{B})$ bond is similar to the Pt-Pt bond strengthening when oxygen atom is adsorbed on fcc site of $\operatorname{Pt}(111)$ surface, while bond weakening that happens on $\operatorname{Pt}\left(\mathrm{c}_{2}\right)-\operatorname{Pt}(\mathrm{B})$ and $\operatorname{Pt}\left(\mathrm{c}_{2}\right)-\operatorname{Pt}\left(\mathrm{A}_{2}\right)$ bonds is equivalent to the case of Pt-Pt bond weakening that is rendered by atomic oxygen adsorption on hcp site. This bond strengthening and weakening phenomenon has been called d-electron frustration by Feibelman ${ }^{18)}$. This proves that Pt-Pt bonds in pure $\mathrm{Pt}$ cluster and $\mathrm{Pt}(111)$ surface react in similar fashion with oxygen, which means the concept of d-electron frustration model is also applicable to $\mathrm{Pt}$ cluster as small as 55-atom. However, Ni-Pt bonds in $\mathrm{Ni}$ @ Pt cluster do not accommodate this model where atomic oxygen adsorption on any site causes the weakening of Ni-Pt bond.

Nonetheless, the analogy of this model can be helpful in explaining why $\mathrm{Ni}\left(\mathrm{c}_{2}\right)-\mathrm{Pt}(\mathrm{B})$ bond is stretched more than twice the bond elongation experienced by $\operatorname{Pt}\left(\mathrm{c}_{2}\right)-\operatorname{Pt}(\mathrm{B})$ bond $(0.38 \AA$ against $0.17 \AA)$. Due to oxygen adsorption, charge transfer from $\mathrm{Pt}(\mathrm{B})$ to oxygen atom leads to increasing repulsive force between the two atoms. To compensate this, some excessive charge of $\mathrm{Pt}(\mathrm{B})-5 \mathrm{~d}$ states atom along the $\mathrm{Pt}(\mathrm{B})-\mathrm{O}$ bond axis are allocated to either $\mathrm{Ni}\left(\mathrm{c}_{2}\right)$ or $\mathrm{Pt}\left(\mathrm{c}_{2}\right)$ atoms. Since most unoccupied $\mathrm{Pt}\left(\mathrm{c}_{2}\right)-5 \mathrm{~d}$ states are anti-bonding states, charge donation from $\mathrm{Pt}(\mathrm{B})$ contributes to bond weakening. For the $\mathrm{Ni}\left(\mathrm{c}_{2}\right)$ atom case, due to $\mathrm{Pt}$ greater electronegativity, it is unlikely for $\mathrm{Pt}(\mathrm{B})$ to donate charge to $\mathrm{Ni}\left(\mathrm{c}_{2}\right)$ atom. Therefore, the $\mathrm{Pt}(\mathrm{B})$ atom only option is to move away as far as possible from $\mathrm{Ni}\left(\mathrm{c}_{2}\right)$ atom. The increasing of anti-bonding profile perpetuated by $\mathrm{Ni}\left(\mathrm{c}_{2}\right)-3 \mathrm{~d}_{\mathrm{xz}}$ states (see Fig. 5) is presumed because $\mathrm{Ni}\left(\mathrm{c}_{2}\right)$ atom interacts with $\mathrm{Pt}(\mathrm{B})$ atom. Similar assumption goes for the $\operatorname{Ni}\left(c_{1}\right)-\operatorname{Pt}\left(A_{1}\right)$ bond in vertex A site. Hence, it could be argued that the stability of $\mathrm{Ni}$ (core)-Pt(shell) bonds in core-shell Ni@Pt cluster is easily disrupted by oxygen than that of $\mathrm{Pt}$ (core)-Pt(shell) bonds in pure $\mathrm{Pt}$ cluster.

As summary, the direct impact of Pt oxide formation on core-shell Ni@Pt cluster in adjusting oxygen adsorption energy will be explained. Previously, it has been shown that core-shell $\mathrm{Ni} @ \mathrm{Pt}$ cluster weakly binds oxygen as compared to pure $\mathrm{Pt}$ cluster because there is strong $3 \mathrm{~d}-5 \mathrm{~d}$ hybridization which reduces the available $\mathrm{Pt}-5 \mathrm{~d}$ states that can interact with O-2p states. When Ni$\mathrm{Pt}$ bonds are weakened, it also means that $3 \mathrm{~d}-5 \mathrm{~d}$ hybridization between $\mathrm{Ni}$ and $\mathrm{Pt}$ atoms is weakened. Thus, it reverses the preceding Pt-5d states shifting to lower energy region and provides more Pt-5d states at higher energy region close to the Fermi level that can form bonding with O-2p states. As the result, the oxygen adsorption energy in core-shell Ni@Pt cluster will increase such as the one observed in bridge site case where the energy resembles the one obtained in pure Pt cluster.

\section{Conclusion}

We have studied the role of alloying and geometry relaxation in determining oxygen adsorption energy in core-shell Ni@Pt cluster. Weaker oxygen adsorption energy in core-shell Ni@Pt is caused by 3d-5d hybridization between $\mathrm{Ni}$ and $\mathrm{Pt}$ atoms, which, reduce the available Pt-5d states that can interact with O-2p states to form stronger Pt-O bonds. However, geometry relaxation in the cluster that is induced by oxygen undermines the effectiveness of core $\mathrm{Ni}$ atoms in rendering shell $\mathrm{Pt}$ atoms to weakly bind oxygen. The oxygen adsorption site preference in both clusters is greatly affected by their geometric structure.

Furthermore, the result shows that $5 \mathrm{~d}-5 \mathrm{~d}$ hybridization of $\mathrm{Pt}$ (core)-Pt(shell) bonds in pure Pt cluster in the presence of oxygen can turn into more attractive or repulsive bonding character depending on the adsorption site of oxygen. In Ni@Pt cluster, the 3d-5d hybridization of Ni-Pt bonds under oxygen presence always developes into repulsive bonding character, hence, weakens the bonding at $\mathrm{Ni} / \mathrm{Pt}$ interface. As consequence, the weakened Ni-Pt bonds will increase Pt-O bonding strength. In general, pure Pt cluster binds oxygen stronger as compared to core-shell Ni@Pt cluster because the cluster also undergoes geometric relaxation that stabilizes Pt-O bonds.

\section{Acknowledgements}

This work is supported in part by MEXT Grant-in-Aid for Scientific Research on Innovative Areas Program (2203-22104008) and Scientific Research programs (A) (24246013); JST ALCA Program "Development of Novel Metal-Air Secondary Battery Based on Fast Oxide Ion Conductor Nano Thickness Film" and Strategic JapanCroatia Research Cooperative Program on Materials Sciences Program "Theoretical Modeling and Simulations of the Structural, Electronic, and Dynamical Properties of Surfaces and Nanostructures in Materials Science Research". Some of the numerical calculations presented here were done using the computer facilities at the following institutes: CMC (Osaka University), ISSP, KEK, NIFS, and YITP.

\section{References}

1) M. Moldovan, M. A. Palacios, M. M. Gómez, G. Morrison, S. Rauch, C. McLeod, R. Ma, S. Caroli, A. Alimonti, F. Petrucci, B. Bocca, P. Schramel, M. Zischka, C. Pettersson, U. Wass, M. Luna, J. C. Saenz and J. Santamaría: Sci. Total Environ., 296 (2002) 199.

2) H. Kishi, F. Oemry, T. Q. Nguyen, S. Kunikata, H. Nakanishi, H. Kasai, H. Maekawa and K. Osumi: Curr. Appl. Phys., 12 (2012) S110.

3) T. Q. Nguyen, M. C. S. Escaño, H. Nakanishi, H. Kasai, H. Maekawa, K. Osumi and K. Sato: Appl. Surf. Sci., 288 (2013) 244.

4) S. Mulla, N. Chen, L. Cumaranatunge, G. Blau, D. Zemlyanov, W. Delgass, W. Epling and F. Ribeiro: J. Catal., 241 (2006) 389.

5) C. Mager-Maury, G. Bonnard, C. Chizallet, P. Sautet and P. Raybaud: Chem. Cat. Chem., 3 (2011) 200.

6) F. C. Galisteo, R. Mariscal, M. L. Granados, M. D. Z. Poves, J. L. G. Fierro, V. Kroger and R. L. Keiski: Appl. Catal. B, 72 (2007) 272.

7) H. Shinjoh: Catal. Surv. Asia, 13 (2009) 184.

8) S. R. de Miguel, I. M. J. Vilella, S. P. Maina, D. San JoséAlonso, M. C. Román-Martínez and M. J. Illán-Gómez: Appl. Catal. A, 435-436 (2012) 10.

9) M. García-Diéguez, E. Finocchio, M. Á. Larrubia, L. J. Alemany and G. Busca: J. Catal., 274 (2010) 11.

10) E. Örücü, F. Gökaliler, A. E. Aksoylu and Z. I. Önsan: Catal. Lett., 120 (2007) 198. 
11) B. S. Çağlayan, Z. İlsen Önsan and A. E. Aksoylu: Catal. Lett., $102(2005) 63$.

12) J. Li, W. P. Tian, X. Wang and L. Shi: Chem. Eng. J., 175 (2011) 417.

13) F. Oemry, H. Nakanishi, H. Kasai, H. Maekawa, K. Osumi and K. Sato: J. Alloys Comp., 594 (2014) 93.

14) A. Boubnov, S. Dahl, E. Johnson, A. P. Molina, S. B. Simonsen, F. M. Cano, S. Helveg, L. J. Lemus-Yegres and J.-D. Grunwaldt: Appl. Catal. B, 126 (2012) 315.

15) P. G. Allen, S. D. Conradson, M. S. Wilson, S. Gottesfel, I. D. Raistrick, J. Valerio and M. Lovato: J. Electroanal. Chem., 384 (1995) 99.

16) H. Imai, K. Izumi, M. Matsumoto, Y. Kubo, K. Kato and Y. Imai: J. Am. Chem. Soc., 131 (2009) 6293.

17) C. Hock, S. Straßburg, H. Haberland, B. V. Issendorff, A. Aguado and M. Schmidt: Phys. Rev. Lett., 101 (2008) 023401.

18) P. Feibelman: Phys. Rev. B, 56 (1997) 10532.
19) J. R. Kitchin, J. K. Nørskov, M. A. Barteau and J. G. Chen: Phys. Rev. Lett., 93 (2004) 156801.

20) J. P. K. Doye and D. J. Wales: New J. Chem., 22 (1998) 733.

21) L.-L. Wang and D. D. Johnson: J. Am. Chem. Soc., 131 (2009) 14023.

22) G. Kresse and J. Hafner: Phys. Rev. B, 47 (1993) 558.

23) G. Kresse and J. Hafner: Phys. Rev. B, 48 (1993) 13115.

24) G. Kresse and J. Hafner: Phys. Rev. B, 49 (1994) 251.

25) J. Perdew, K. Burke and M. Ernzerhof: Phys. Rev. Lett., 77 (1996) 3865.

26) S. Mukerjee, S. Srinivasan, M. P. Soriaga and J. McBreen: J. Phys. Chem., 99 (1995) 4577.

27) M. Teliska, V. S. Murthi, S. Mukerjee and D. E. Ramaker: J. Electrochem. Soc., 152 (2005) A2159.

28) C. Lu and G. Henkelman: J. Phys. Chem. Lett., 2 (2011) 1237.

29) B. Han, C. Miranda and G. Ceder: Phys. Rev. B, 77 (2008) 075410 . 